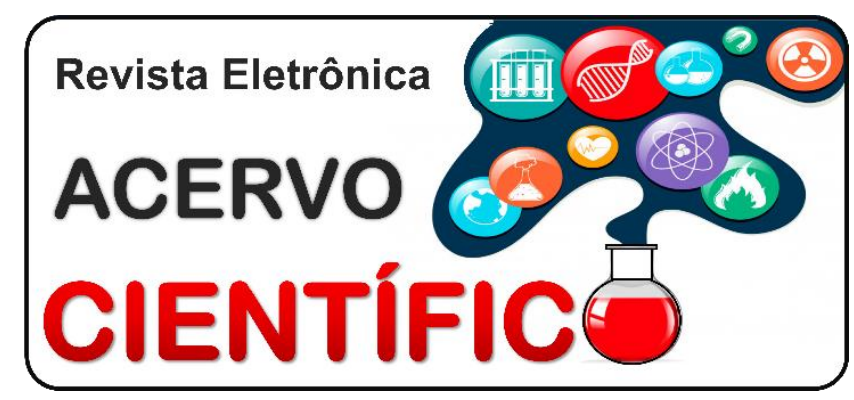

ARTIGO ORIGINAL

Recebido em: 5/2020

Aceito em: 6/2020

Publicado em: $8 / 2020$

\title{
Hipertensão arterial sistêmica: prevalência da adesão ao tratamento em uma unidade de saúde do Pará
}

\author{
Systemic arterial hypertension: prevalence of adherence to treatment in a health unit of \\ Pará
}

Hipertensión arterial sistémica: prevalencia de adherencia al tratamiento en una unidad de
salud de Pará

Karen Regina Guimarães Dantas ${ }^{1 *}$, Bruno da Silva Quaresma ${ }^{1}$, Fernanda Moema Mendes Leite ${ }^{1}$, Gabriela Rosa Bernardo ${ }^{1}$. Afonso Vinicius de Lima Filgueira ${ }^{1}$, Rafael Schalcher Martins e Silva ${ }^{2}$, Renata Ogawa Furtado Rodrigues ${ }^{1}$, Mariana Rosa Bernardo ${ }^{1}$, Fernanda Vidal Pacheco ${ }^{1}$, Osvaldo da Silva Peixoto 1 .

\begin{abstract}
Resumo: Estabelecer a prevalência de adesão ao tratamento da Hipertensão Arterial Sistêmica (HAS) na área de uma estratégia saúde da família no Pará. Estudo quantitativo, transversal e de caráter retrospectivo sobre as características e o perfil dos pacientes portadores de HAS que aderiram ao tratamento, realizado através da observância e coleta dos dados de elementos preenchidos previamente nos prontuários da unidade em questão, respondendo às questões levantadas no questionário individual. Foram avaliados 62 participantes, sendo $66,1 \%$ mulheres, $64,5 \%$ idosos. $35,5 \%$ Eutróficos, $64,5 \%$ acima do peso normal, 45,2 classificados como Hipertensos, 32,3\% com Hipertensão Estágio 1, 71\% fazem uso de medicamentos, 47,7\% utilizavam Losartana, e 62,9\% abandonaram o tratamento. Assim, foi possível estabelecer e correlacionar esses dados com informações de outros estudos, possibilitando estabelecer dentre os participantes características que corroboraram para a adesão ou abandono do tratamento e qual o perfil desses indivíduos. A prevalência de adesão ao tratamento, dos participantes, com hipertensão arterial sistêmica são indivíduos do sexo feminino, com mais de 60 anos, que apresentam hipertensão, Índice de Massa Corporal (IMC) acima do peso normal, fazem uso de medicamentos, principalmente, uma vez ao dia, sendo os mais utilizados o Losartana, Hidroclorotiazida e Captropil.
\end{abstract}

Palavras-chave: Hipertensão, Epidemiologia, Atenção básica à saúde.

\begin{abstract}
To establish the prevalence of adherence to the treatment of Systemic Arterial Hypertension (SAH) in the area of a family health strategy in Pará. Quantitative, cross-sectional and retrospective study on the characteristics and profile of patients with SAH who adhered to the treatment, carried out through observing and collecting data from elements previously filled in the medical records of the unit in question, answering the questions raised in the individual questionnaire. 62 participants were evaluated, $66.1 \%$ women, $64.5 \%$ elderly, 35.5\% Eutrophic, 64.5\% overweight, 45.2 classified as Hypertensive, 32.3\% with Stage 1 Hypertension, $71 \%$ use medication, $47.7 \%$ used Losartana, and $62.9 \%$ abandoned treatment. Thus, it was possible to establish and correlate these data with information from other studies, making it possible to establish among the participants characteristics that corroborated the adherence or abandonment of the treatment and the profile of these individuals. The prevalence of adherence to treatment, of the participants, with systemic arterial hypertension are female individuals, over 60 years old, who present hypertension, BMI above normal weight,
\end{abstract}

1 Centro Universitário Metropolitano da Amazônia (UNIFAMAZ), Belém - Pará.

*E-mail: karengdantas@hotmail.com

${ }^{2}$ Centro Universitário do Planalto Central Aparecido dos Santos (UNICEPLAC), Brasília - DF. 
make use of medicines, mainly, once a day, being the most used are Losartana, Hydrochlorothiazide and Captropil.

Keywords: Hypertension, Epidemiology, Primmary health care.

Resumen: Establecer la prevalencia de adherencia al tratamiento de la hipertensión arterial sistémica (HSA) en el área de una estrategia de salud familiar en Pará. Estudio cuantitativo, transversal y retrospectivo sobre las características y el perfil de los pacientes con HSA que se adhirieron al tratamiento, realizado a través de la observación y la recopilación de datos de elementos previamente completados en los registros médicos de la unidad en cuestión, respondiendo las preguntas formuladas en el cuestionario individual. Se evaluaron 62 participantes, $66.1 \%$ mujeres, $64.5 \%$ ancianos, $35.5 \%$ eutróficos, $64.5 \%$ sobrepeso, 45.2 clasificados como hipertensos, $32.3 \%$ con hipertensión en etapa $1,71 \%$ usa medicamentos, $47.7 \%$ usa Losartana y $62.9 \%$ abandona el tratamiento. Por lo tanto, fue posible establecer y correlacionar estos datos con información de otros estudios, lo que permitió establecer entre los participantes características que corroboraron la adherencia o el abandono del tratamiento y el perfil de estos individuos. La prevalencia de adherencia al tratamiento, de los participantes, con hipertensión arterial sistémica son mujeres, mayores de 60 años, que presentan hipertensión, IMC por encima del peso normal, utilizan medicamentos, principalmente, una vez al día, siendo el Los más utilizados son Losartana, Hidroclorotiazida y Captropil.

Palabras clave: Hipertensión, Epidemiología, Atención primaria de salud.

\section{INTRODUÇÃO}

A hipertensão arterial sistêmica (HAS) é uma condição clínica multifatorial e o principal fator de risco para desenvolvimento de doenças cardiovasculares, cerebrovasculares e renais. Segundo dados da Organização Pan-Americana de Saúde, globalmente, estima-se que 18\% das mortes, ou seja, 9,4 milhões, foram atribuídas ao aumento da pressão arterial em 2010. Cerca de 4 em cada 10 adultos com mais de 25 anos de idade tem hipertensão, e em muitos países 1 em cada 5 pessoas tem pré-hipertensão. Esse fato, portanto, caracterizaa como uma das maiores causas de redução da expectativa e da qualidade de vida dos indivíduos (ORGANIZAÇÃO PAN-AMERICANA DA SAÚDE, 2016).

Na região das Américas, a hipertensão afeta entre 20-40\% da população adulta da região, o que significa que cerca de 250 milhões de pessoas sofrem com a HAS. Ademais, ocorre 1,6 milhões de mortes, a cada ano, causadas por doenças cardiovasculares, dessas cerca de meio milhão ocorrem em pessoas com menos de 70 anos de idade, o que é considerado morte prematura e evitável. A partir dessas perspectivas é importante frisar que a HAS está enquadrada como um grave problema de saúde pública no Brasil. De tal forma que sua prevalência no território varia entre $22 \%$ e $44 \%$ para adultos, ultrapassa os $50 \%$ para faixa etária entre 60 a 69 anos e para indivíduos acima de 70 anos alcança 75\% (BRASIL, 2013; SOCIEDADE BRASILEIRA DE CARDIOLOGIA, 2014). A Hipertensão Arterial Sistêmica pode ser dividida em estágios: normal sendo pressão sistólica menor que 130 e diastólica menor que 85, hipertensão grau I de 140-159 na pressão sistólica e 90-99 na diastólica, hipertensão grau II de 160-169 na sistólica e 100-109 na diastólica e a hipertensão grau III são valores maiores que 169 na pressão sistólica e maior que 109 na diastólica (DÓREA EL, et al., 2004).

Além disso, inúmeros estudos têm apontado a enorme dificuldade vivida pelas pessoas com hipertensão para persistirem seguindo as recomendações médicas, com expressiva frequência de abandono do tratamento. Estima-se que cerca de dois terços dos pacientes com Hipertensão Arterial (HA) não têm seus níveis pressóricos adequados, devido, em grande parte, ao seguimento incorreto do tratamento medicamentoso (BRASIL, 2011). Estudos internacionais e nacionais mostram grande variação nas taxas de adesão e abandono observadas, por exemplo, no Brasil, cerca de $50 \%$ dos hipertensos abandonam o tratamento no primeiro ano em que são submetidos a acompanhamento médico e, após cinco anos, apenas $17 \%$ permaneceram em tratamento (MALACHIAS MVB, et al., 2016). Dessa forma, a continuidade da doença permanece e os problemas a ela ligados são cada vez mais evidentes. Essa variação deve-se a vários fatores, entre eles o método de medida utilizado, o ponto de corte adotado para a definição de adesão e a seleção da amostra estudada. É importante, também, reconhecer que a assistência a pessoas com doenças crônicas, 
como a HA, o diabetes e outras, requer considerar a complexidade do cuidado (e do autocuidado) em condições de cronicidade. Destaca-se, nesse reconhecimento, a crítica à abordagem estritamente técnica da adesão do paciente e à restrita consideração das dificuldades vividas em seu cotidiano (DÓREA EL, et al., 2004).

Mediante esse panorama, o Ministério da Saúde desenvolveu a plataforma HIPERDIA, Sistema de Cadastramento e Acompanhamento de Hipertensos e Diabéticos, a qual tem a finalidade de cadastrar e acompanhar os portadores de hipertensão arterial e/ou diabetes mellitus atendidos na rede ambulatorial do Sistema Único de Saúde-SUS, e isso possui uma relevância por permitir gerar informação para aquisição, dispensação e distribuição de medicamentos de forma regular e sistemática a todos os pacientes cadastrados além de organizar os pacientes para desenvolvimento de pesquisas como esta (LIMA TM, et al., 2010).

O objetivo da presente pesquisa visa estabelecer a prevalência de adesão ao tratamento da Hipertensão Arterial Sistêmica na área da Estratégia Saúde da Família de uma cidade do Pará, entre os anos de janeiro de 2012 a dezembro de 2014.

\section{MÉTODOS}

A referida pesquisa tem o caráter de um estudo quantitativo, transversal e retrospectivo sobre as características dos pacientes portadores de Hipertensão Arterial Sistêmica assistidos por uma Estratégia Saúde da Família de uma cidade do Pará.

Este projeto foi aprovado pelo Comitê de Ética em Pesquisa (CEP) sob número de parecer 2.324.703. Os participantes foram estudados segundo os preceitos da Declaração de Helsinque e do Código de Nuremberg, seguindo as determinações da resolução 466/2012 do Conselho Nacional de Saúde, cumprindo-se as orientações descritas no Termo de Consentimento Livre e Esclarecido (TCLE).

. A coleta de informações ocorreu por meio da avaliação criteriosa do perfil dos participantes que aderiram ao tratamento através da observância e coleta dos dados contidos nos prontuários da unidade em questão, respondendo às questões levantadas no questionário individual, no qual foram abordadas informações pessoais como sexo, idade, índice de massa corporal, uso, frequência e quais os medicamentos; além de se abandonou ou não o tratamento.

Neste estudo, foram incluídos indivíduos, de ambos os sexos, a partir de 18 anos, que apresentem Hipertensão Arterial Sistêmica, cadastrados na Estratégia Saúde da Família de uma cidade do Pará no período de Janeiro de 2012 a Dezembro de 2014, cujos respectivos prontuários esteavam devidamente preenchidos e atualizados e que concordaram em colaborar com a pesquisa mediante a assinatura do Termo de Consentimento Livre e Esclarecido (TCLE). Foram excluídos aqueles que apresentaram prontuário com letra ilegível, e/ou sem identificação. Assim como, indivíduos que possuíam informações incompletas em seus prontuários.

Foram coletadas informações dos indivíduos participantes da pesquisa, segundo o cálculo amostral, utilizando como instrumento da coleta de dados os prontuários que possibilitaram, subsequentemente, o preenchimento do questionário com os elementos de interesse, como idade, sexo, índice de massa corporal, os tipos de tratamentos que foram submetidos e a adesão a ele.

Os dados referentes às características clínicas foram tratados utilizando estatística descritiva, expressos sob a forma de Média, Desvio Padrão, Mediana, Percentis e frequências absoluta e relativa conforme o caso e representados em tabelas e/ou gráficos. A diferença entre as proporções em cada categoria observada será avaliada utilizando o teste do Qui-Quadrado ou teste G (Aderência), conforme a necessidade.

\section{RESULTADOS}

Por meio dos dados analisados foi possível encontrar que a mostra de distribuição dos usuários hipertensos possui $66,1 \%$ do sexo feminino $(n=41)$ e $33,9 \%$ do sexo masculino $(n=21)$, sendo esse dado significativamente estatístico $(p=0,0158)$. Além disso, foi encontrado a distribuição por faixa etária de $64,5 \%$ 
$(n=40)$ terem mais de 60 anos, 30,6\% $(n=19)$ terem entre 50-59 anos e apenas 4,8\% $(n=3)$ tinham menos de 50 anos, sendo este dado com relevância estatística $(p<0,0001)$ pelo teste Qui Quadrado de Aderência.

Além disso, foi analisado de acordo com a classificação nutricional evidenciando que a maioria é eutrófica $(35,5 \%-n=22)$ ou possui sobrepeso $(33,9 \%-n=21)$, sendo que o restante está com obesidade grau I $(22,6 \%$ - $n=14)$ e obesidade grau II $(8,1 \%-n=5)(p=0,8)$. Este dado foi analisado sob a ótica de divisão de peso normal $(35,5 \%-n=22)$ e acima do peso $(64,5 \%-n=40)$ sendo este dado significativo $(p<0,0001)$.

Posteriormente, os pacientes foram avaliados conforme o estágio de Hipertensão, sendo a maioria enquadrada em Hipertensão Estágio 1 (32,3\%) e Ótima Pressão Arterial (30,6\%) (Tabela 1).

Tabela 1 - Mostra a distribuição dos usuários hipertensos da Unidade de Saúde da segundo a pressão arterial entre $2012-2014$.

\begin{tabular}{ccc}
\hline Pressão Arterial & Pacientes & $\%$ \\
\hline Ótima & 19 & $30,6 \%$ \\
Normal & 15 & $24,2 \%$ \\
Hipertensão Estágio 1 & 20 & $32,3 \%$ \\
Hipertensão Estágio 2 & 4 & $6,5 \%$ \\
Hipertensão Estágio 3 & 4 & $6,5 \%$ \\
\hline Total & $\mathbf{6 2}$ & $\mathbf{1 0 0 , 0} \%$ \\
\hline
\end{tabular}

Legenda: ${ }^{*} p=0.0005$ Teste Qui-Quadrado Aderência.

Fonte: Dantas KRG, et al., 2020.

A maioria dos pacientes hipertensos $(71 \%-n=44)$ também fazem uso de alguma medicação para controle da pressão arterial e 29\% ( $n=18$ ) não utilizam medicação para controle (Tabela 2).

Tabela 2 - Mostra a distribuição dos usuários hipertensos da Unidade de Saúde da segundo o uso de medicamentos entre $2012-2014$.

\begin{tabular}{ccc}
\hline Faz uso de medicamentos & Pacientes & $\%$ \\
\hline Sim $^{*}$ & 44 & $71,0 \%$ \\
Não & 18 & $29,0 \%$ \\
\hline Total & $\mathbf{6 2}$ & $\mathbf{1 0 0 , 0 \%}$
\end{tabular}

Legenda: ${ }^{*} p=0.0015$ Teste Qui-Quadrado Aderência.

Fonte: Dantas KRG, et al., 2020.

A Tabela 3 mostra a distribuição de usuários conforme frequência de utilização de medicamentos para controle da pressão arterial, sendo que $59,1 \%$ utiliza uma vez ao dia, $29,5 \%$ duas vezes ao dia e o restante três ou mais vezes ao dia.

Tabela 3 - Distribuição dos usuários hipertensos da Unidade de Saúde da Família segundo a frequência de medicamentos entre $2012-2014$.

\begin{tabular}{ccc}
\hline Frequência de medicamentos & Pacientes & $\%$ \\
\hline Uma vez ao dia** & 26 & $59,1 \%$ \\
Duas vezes ao dia & 13 & $29,5 \%$ \\
Três ou mais vezes ao dia & 5 & $11,4 \%$ \\
\hline Total & $\mathbf{4 4}$ & $\mathbf{1 0 0 , 0 \%}$
\end{tabular}

Legenda: ${ }^{*} \mathrm{p}=0.0015$ Teste Qui-Quadrado Aderência

Fonte: Dantas KRG, et al., 2020.

Já a distribuição de medicamentos utilizados pelos pacientes encontra distribuição segundo Tabela 4 abaixo. A maioria utiliza Losartana (47,7\%) e Hidroclorotiazida (27,3\%). 
Tabela 4 - Mostra a distribuição dos usuários hipertensos da Unidade de Saúde da Família segundo os medicamentos utilizados entre $2012-2014$.

\begin{tabular}{ccc}
\hline Medicamentos utilizados & Pacientes & $\%$ \\
\hline Losartana & 21 & $47,7 \%$ \\
Hidroclorotiazida & 12 & $27,3 \%$ \\
Capitopril & 8 & $18,2 \%$ \\
Acido Acetilsalicílico (AAS) & 7 & $15,9 \%$ \\
Sinvastatina & 7 & $15,9 \%$ \\
Atenolol & 5 & $11,4 \%$ \\
Nifedipina & 4 & $9,1 \%$ \\
Propranolol & 2 & $4,5 \%$ \\
Pressat & 1 & $2,3 \%$ \\
\hline
\end{tabular}

Fonte: Dantas KRG, et al., 2020.

Foram encontrados resultados de abandono de tratamento sendo a maioria, 62,9\% ( $n=39)$ relatar já ter abandonado em algum momento e o restante nega a interrupção do tratamento, sendo este dado significativo $(p=0,0422)$. A distribuição dos medicamentos por sexo também foi analisada, caracterizando que $75,6 \%$ do público feminino e $61,9 \%$ do masculino utilizam medicações para controle da PA.

Já evidenciando a classificação de hipertensão por sexo, também foi encontrado que na classificação Ótima PA a maioria são do sexo feminino e em categoria Hipertensão sobressai o sexo masculino.

Por meio desta pesquisa foi possível encontrar a distribuição de pacientes segundo classificação de IMC comparando com sexo, encontrando então que a maioria dos pacientes da classificação Eutrófica são do sexo feminino e na classificação Sobrepeso e Obesidade a maioria são do sexo masculino. Por fim, foi encontrado distribuição igualitária na comparação de sexo com abandono de tratamento, encontrando que não possui um sexo com maior prevalência de abandono.

\section{DISCUSSÃO}

A hipertensão arterial sistêmica (HAS) é uma das doenças mais prevalentes no mundo, com taxas que superam $30 \%$. Mudanças sociais, econômicas e demográficas ocorridas no Brasil, no processo denominado transição epidemiológica, resultaram em aumento considerável da morbidade e da mortalidade por doenças crônicas não-transmissíveis, entre as quais a HAS é a maior representante (DUARTE MTC, et al., 2010).

Este presente estudo revela que dos 62 participantes estudados, $41(66,1 \%)$ eram do sexo feminino e 21 $(33,9 \%)$ do sexo masculino. Corroborando com achados de outras investigações realizadas, e observou-se associação estatisticamente significativa entre os sexos, ao contrário dos resultados encontrados em um estudo de HAS como fator de risco para doenças cardiovasculares. A quantidade elevada de mulheres em tratamento na unidade de saúde parte da hipótese de serem mulheres com disponibilidade de tempo maior, uma vez que a quantidade de mulheres apenas cuidadoras do lar é alta na região da Estratégia Saúde da Família (ESF), além do fato, das mulheres procurarem a unidade para rastreio de doenças ginecológicas e pelo oportunismo, identificarem o acometimento da HAS (BORGES CP, et al., 2006).

Em relação à faixa etária, a análise de dados mostrou que $64,5 \%$ da população estudada têm 60 anos ou mais. Resultado com incidência significante e que se assemelha ao estudo sobre Prevalência, Consciência, Tratamento e Influência de Variáveis Socioeconômicas sobre controle da pressão arterial, o qual revelou que a faixa etária com maior número de hipertensos é entre 65 a 74 anos. Vale ressaltar que é considerável a identificação da idade prevalente, uma vez que, as elevações da pressão sanguínea em pessoas com mais de 60 anos é $60 \%$ maior. Isso se deve ao fato das alterações fisiológicas ocorridas com o aumento da idade, como a deposição de cálcio nos vasos sanguíneos que vão enrijecendo, estreitando a luz do lúmen e aumentando a pressão. Vale considerar que como a maioria dos pacientes cadastrados são mulheres, podese também relacionar a idade superior a 60 anos com a menopausa, uma vez que a queda do estrógeno torna o endotélio mais vulnerável, deixando de produzir substâncias que levaria a dilatação dos vãos, ocasionando a Hipertensão (BRASIL, 2012). 
Já para outra variável, como a classificação nutricional, nota-se que $35,5 \%$ dos participantes possuíam IMC Eutrófico, o que diverge do estudo que relaciona fatores associados em Homens e Mulheres residentes em municípios da Amazônia Legal, que revelou uma associação três vezes maior em participantes obesos. Entretanto, em outra perspectiva, quando se relaciona apenas peso normal e acima do peso normal - que inclui as classificações sobrepeso, obesidade grau 1 e obesidade grau 2 - fica evidente que a porcentagem de indivíduos acima do peso normal é maior $(64,5 \%)$, e possui incidência significativa. Condizente dessa forma, com o estudo epidemiológicos realizado no Centro Oeste, os quais tem mostrado a prevalência de hipertensão arterial mais elevada em indivíduos com peso acima do normal, do que naqueles dentro da faixa de peso normal. É importante considerar a classificação nutricional pois, o aumento de peso se relaciona com a hipertensão por aumentar os níveis de insulina no sangue, retenção de sódio pelos rins, e ainda, que essa associação - HAS e obesidade - pode ocasionar uma Síndrome Metabólica, faltando apenas mais um critério para o diagnóstico da mesma (SOCIEDADE BRASILEIRA DE CARDIOLOGIA, 2011).

Ainda no quesito IMC, é importante relacionar a classificação nutricional com o sexo. Observou-se nessa pesquisa que os indivíduos com classificação Eutrófica são de prevalência do sexo feminino (43,9\%), e de classificação sobrepeso $(42,9 \%)$ e obesidade $(31,9 \%)$ do sexo masculino, toda via, sem incidência significante. Sendo que esses dados diferem parcialmente dos obtidos em estudos realizados pelo Hospital das Clínicas de Goiás, os quais apresentaram maior sobrepeso em homens do que em mulheres, e o sexo feminino por sua vez, teve maior obesidade quando comparados com o sexo oposto (SOCIEDADE BRASILEIRA DE GERIATRIA, 2011).

No que diz respeito à última aferição de Pressão Arterial (Tabela 6) dos 62 participantes, 19 possuíam a PA ótima (30,6\%), 15 a PA normal $(24,2 \%)$ e 28 a classificação Hipertensão $(45,2 \%)$ - desses, houve divisão em estágio 1, 2 e 3 . Havendo assim, associação significativa, evidenciando que o controle da pressão arterial não foi efetivo. Achado semelhante ao artigo Prevalência, reconhecimento e controle da hipertensão arterial sistêmica no estado do Rio Grande do Sul que expôs que cerca de $80 \%$ dos indivíduos estavam na classificação Hipertensão, e somente $25,6 \%$ atingiam controle dos seus níveis pressóricos. Esse achado desperta para as consequências desse descontrole dos níveis de PA, uma vez que pode aumentar os casos de morbidades como Insuficiência Cardíaca, Doenças Coronarianas, e Acidente Vascular Cerebral (RADONAVIC CAT, et al., 2014).

Corroborando com essa visão, é substancial a associação entre a classificação da pressão arterial com o sexo dos indivíduos. Os dados coletados revelaram que na classificação ótima a maioria é do sexo feminino, $(34,1 \%)$ e na classificação de Hipertensão sobressaiu-se o sexo masculino $(47,6 \%)$, apesar de não haver diferença significante. Esse desfecho se assemelha ao trabalho $O$ Controle da Hipertensão Arterial em Mulheres e Homens. Tal resultado se dá por elas possuírem uma percepção mais acurada de sua condição de saúde e, dessa forma, não somente procuram mais os serviços de saúde, bem como tendem a seguir os tratamentos propostos (MARTINS AL, et al., 2001; CHOR D, et al., 2015).

Nesse contexto, houve a análise dos medicamentos utilizados e se utilizados. Observou-se um predomínio de participantes que utilizavam medicação, correspondendo a $71 \%$. Condizente com a pesquisa sobre Acesso e uso de medicamentos para hipertensão arterial no Brasil que expôs que $94,6 \%$ dos pacientes seguiam a prescrição médica com o uso regular da medicação. Quando se relacionou o sexo e utilização do medicamento, $75,6 \%$ dos pacientes femininos e $61,9 \%$ dos pacientes masculinos eram adeptos á medicação, entretanto não houve diferença significante no uso de medicamentos entre os sexos. Descoberta concordante com o Programa HIPERDIA de uma cidade da região sul do país, que mostrou que $37,6 \%$ das mulheres estavam com a pressão arterial controlada, contra $25 \%$ dos homens (MENDES R, 2008; SILVA EC, et al., 2016). A frequência de medicação mais adeptas dos hipertensos foi a de uma vez ao dia, correspondendo a $59,1 \%$, com incidência significante. Deve-se considerar que isso se dá tanto pela prescrição médica quanto pela escolha do paciente em como progredir o tratamento, uma vez que opta muitas vezes em não seguir a risca, e ter autonomia em usar a medicação apenas às vezes que achar necessário.

Sobre os medicamentos utilizados, constataram-se no estudo que o fármaco de primeira escolha na unidade foi o BRA's (Bloqueador de Receptor de Angiotensina II) como a Losartana (47,7\%), em segundo os 
Diuréticos como a Hidroclorotiazida (27,3\%) e em terceiro os Inibidores da ECA como o Captopril (18,2\%). Dessa forma, tais dados discordam do estudo sobre Perfil da Terapêutica Utilizada em Pacientes Hipertensos atendidos no Hospital de Urgência de Goiânia, que revelou que os IECA's são prescritos com mais frequência, seguidos dos diuréticos e betabloqueadores (NASCENTE FMN, et al., 2009). Deve-se ressaltar, que também foi constatado a utilização de outros medicamentos, contudo, em menor porcentagem como o ácido Acetilsalicílico (15,9\%), Sinvastatina (15,9\%), Atenolol (11,4\%), Nifedipina (9,1\%), Propranolol (4,5\%) e Pressat (2,3\%).

Quanto ao abandono do tratamento, houve incidência significante de pacientes que não mantiveram o tratamento, logo, dos 62 participantes: 39 não deram continuidade (62,9\%) e apenas 23 continuaram regularmente indo à ESF (37,1\%). Ao contrário da pesquisa realizada pelo autor Pereira QTS (2011), na Bahia, onde $63,8 \%$ seguiam regularmente o tratamento, e $36,2 \%$ não seguiam.

Nessa perspectiva, ao observar a relação do abandono do tratamento com o sexo, foi visto que dos pacientes femininos $56,1 \%$ não mantiveram e $43,9 \%$ prosseguiram. Já os pacientes masculinos $76,2 \%$ não mantiveram o tratamento e $23,8 \%$ mantiveram. Contudo, sem diferença estatisticamente relevante, embora os dados transpareçam a possibilidade de existir complicação para permanência no tratamento. Segundo o artigo do autor Pereira QTS, dentre as principais causas estão a falta de medicamentos $26,5 \%$, a falta de instruções quanto ao tratamento não medicamentoso $18,4 \%$, demora ou não atendimento dos pacientes $37,1 \%$ e inadequação da relação profissional de saúde-paciente $18 \%$ na PSF, isso faz com que os motivos relacionados acima se tornem mais concretos (PEREIRA QTS, et al., 2011).

A partir dos dados levantados nesse trabalho e diante da realidade assinalada são necessárias à implantação e implementação de ações de promoção da saúde, dirigidas para a educação em saúde e práticas preventivas. Com a finalidade de promover o conhecimento da população sobre a importância do controle da hipertensão arterial; garantir o acesso dos hipertensos a serviços básicos de saúde com resolubilidade e incentivar políticas e programas comunitários. Ressaltando a importância de uma equipe multiprofissional no controle e prevenção da HAS, principalmente em relação aos fatores extrínsecos, modificantes.

\section{CONCLUSÃO}

O presente estudo concluiu que a prevalência de adesão ao tratamento dos participantes, com hipertensão arterial sistêmica na unidade é caracterizado em sua maioria: por indivíduos do sexo feminino, com mais de 60 anos, que apresentam hipertensão, IMC acima do peso normal, fazem uso de medicamentos, principalmente, uma vez ao dia, sendo os mais utilizados o Losartana, Hidroclorotiazida e Capitropil. Ademais, não foram identificadas outras formas de tratamento além da medicamentosa. Ainda deve ser ressaltado que houve um elevado abandono do tratamento. Dessa forma este estudo contribuiu para encontrar um perfil de hipertensos a fim de servir de base para o desenvolvimento de futuras pesquisas e criação de ações em saúde para público direcionado. A principal limitação deste estudo foi o não preenchimento de diversas informações consideradas relevantes nos prontuários da unidade.

\section{REFERÊNCIAS}

1. BORGES CP, Arantes RL. Aspectos epidemiológicos da aderência ao tratamento de hipertensão arterial sistêmica. Revista Médica Ana Costa, Santos. 2006; 11:1.

2. BRASIL. Ministério da Saúde (Brasil). Secretaria de Atenção à Saúde. Departamento de Atenção Básica. Cadernos de Atenção Básica, $n \cong$ 37. Estratégias para o cuidado da pessoa com doença crônica: hipertensão arterial sistêmica. Brasília: Ministério da Saúde, 2013. p. 21-23.

3. BRASIL. Ministério da Saúde. Hiperdia sistema de cadastramento e acompanhamento de hipertensos e diabéticos. 2012.

4. BRASIL. Ministério da Saúde. Portal da saúde, acesso dia 27 de março de 2017. Disponível: www.saude.gov.br.

5. BRASIL. São Paulo (Estado) Secretaria de Saúde. Gabinete do secretário. Assessoria técnica. Manual de orientação clínica: hipertensão arterial sistêmica (HAS)/ Aparecida TerukoTominaga Yamada /Carmen Lavras e Maris Salete Demuner (orgs). São Paulo: SES/SP, 2011. 68 p.; tab; graf. 
6. CHOR D, et al. Prevalence, awareness, treatment and influence of socioeconomic variables on control of high blood pressure: results of the ELSA-Brasil Study. PLOS One. 2015;10(6): e0127382.

7. DÓREA EL, et al. Epidemiologia da hipertensão arterial sistêmica. Hipertensão. 2004; 7:86-9.

8. DUARTE MTC, et al. Motivos do abandono do seguimento médico no cuidado a portadores de hipertensão arterial: a perspectiva do sujeito. Ciênc. saúde coletiva, Rio de Janeiro, 2010, 15(5):10-15.

9. LIMA TM, et al. Perfil de adesão ao tratamento de pacientes hipertensos atendidos na Unidade Municipal de Saúde de Fátima, em Belém, Pará, Amazônia, Brasil. Rev. Pan-Amaz Saúde. 2010; 1:2.

10. MALACHIAS MVB, et al. 7ª Diretriz Brasileira de Hipertensão Arterial. Arq. Bras. Cardiol. 2016; 107(3Supl.3): 1-83.

11. MARTINS AL, et al. Análise da composição corporal e do índice de massa corporal de indivíduos de 18 a 50 anos. Cad Saúde Pública. 2001; 9 (2): 97-110.

12. MENDES R, et al. Envelhecimento e pressão arterial. Acta Med Port. 2008; 21 (2): 193-98.

13. NASCENTE FMN, et al. Hipertensão arterial e sua associação com índices antropométricos em adultos de uma cidade de pequeno porte do interior do brasil. Rev Assoc Med Bras 2009; 55(6): 716 - 722.

14. ORGANIZAÇÃO PAN-AMERICANA DA SAÚDE. Dia mundial da hipertensão 2016, acesso em 03 de novembro de 2016.

15. PEREIRA QTS, et al. Motivos que levam o paciente hipertenso á abandonar o tratamento anti-hipertensivo em uma unidade de saúde. Faculdade Adventista. Bahia, 2011.

16. RADANOVIC CAT, et al. Hipertensão arterial e outros fatores de risco associados às doenças cardiovasculares em adultos. Rev. Latino-Am. Enfermagem jul.-ago. 2014; 22(4): 547-53.

17. SILVA EC, et al. Prevalência de hipertensão arterial sistêmica e fatores associados em homens e mulheres residentes em municípios da Amazônia Legal. Rev bras epidemiol jan-mar 2016; 19 (1): 38-51.

18. SOCIEDADE BRASILEIRA DE CARDIOLOGIA. Revista Brasileira de Hipertensão. 2014; 23 (2): 61-126.

19. SOCIEDADE BRASILEIRA DE CARDIOLOGIA. Revista Brasileira de Hipertensão. $2011 ; 12$ (1): 1 - 42.

20. SOCIEDADE BRASILEIRA DE GERIATRIA. Revista brasileira de Geriatria e Gerontologia. $2011 ; 14$ (4): 271 - 284. 